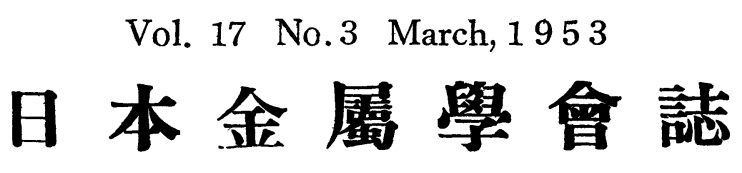

第 17 卷 第 3 號

論 交

\title{
Primary Recrystallization Texture of Copper and Composite Dislocation of Face Centered Cubic Metals*
}

\author{
Isao GoKYu and Hideo ABE \\ Department of Metallurgy, Faculty of Engineering, Tokyo University.
}

(Received October 27,1952 )

Synopsis

Primary recrystallization textures of copper sheets were examined by using X-ray and etching figure methods. $22^{\circ}$ and $38^{\circ}$ rotations around [111] axis and $11^{\circ}$ rotation around [100] axis were found. The [111] rotations can be rationally interpreted by the oriented nucleation in a composite dislocation proposed by the authors.

\section{Experiment}

A $1.5 \mathrm{~cm}$ thick slab was cut from a high purity copper ingot and cold-rolled to a sheet about $0.15 \mathrm{~mm}$ thick. X-ray photograms of this final sheet indicated the existence of two sets of orientation, namely (110)-[112] and (112)-[111]. One of the X-ray photograms of this cold-rolled sheet is as shown in Photo. 1.

Individual specimens of this material about $1.5 \times 2 \mathrm{~cm}$ were used for all heat R.D

$\uparrow$

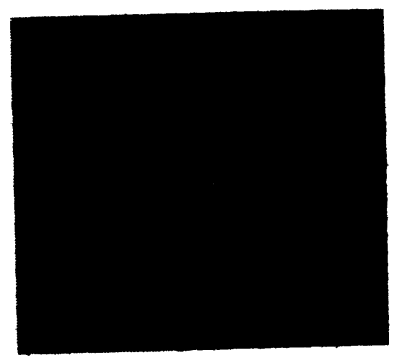

Photo. 1 C X-ray Photogram of Cold-rolled Copper Sheet Subjected a Total Reduction of $99.9 \%$.

$\mathrm{X}$-ray used in this investigation is Mo-Ko ray. $\mathrm{X}$-ray beam is normal to the plane of the specimen.
R. D.

$\uparrow$

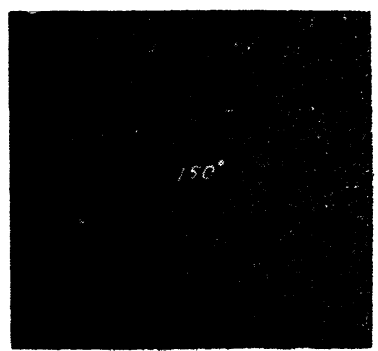

Photo. 2
R.D.

$\uparrow$

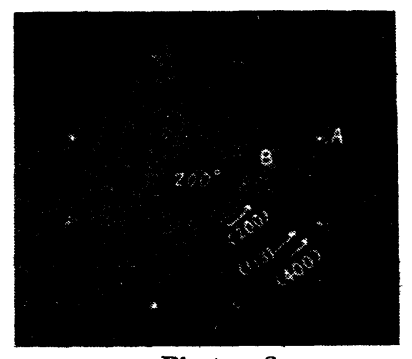

Photo. 3

treatments. The annealings were carried out for $10 \mathrm{~min}$ at $150^{\circ}, 200^{\circ}, 400^{\circ}, 600^{\circ}$ and $800^{\circ}$ Representative $\mathrm{X}$-ray photograms of these annealed specimens are as shown in Photo. 2 5. From these $X$-ray photograms, it was clarified that the main texture of these recrystallized specimens was a cubically

* This paper was read before the Kyushu Univ. Meeting of the Japan Institute of Metals held on November 3rd, 1952. 
aligned one, namely (100)-[001], but other three sets of orientation were found to coexist. And it was readily seen that these three sets of orientation could be brought R.D.

$\uparrow$

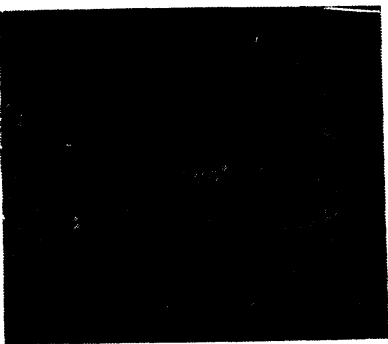

Photo. 4 R.p.

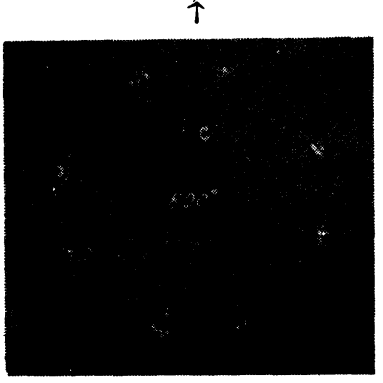

Photo. 5

Photo. 2 5 X-ray Photograms of 99.9\% Cold-rolled and Annealed Specimens. Annealing Temperature is Respectively $150^{\circ}$ (Photo. 2), $250^{\circ}$ (Photo. 3), 400 (Photo. 4) and $600^{\circ}$ (Photo. 5). X-ray Beam is Normal to the Plane of the Specimens.

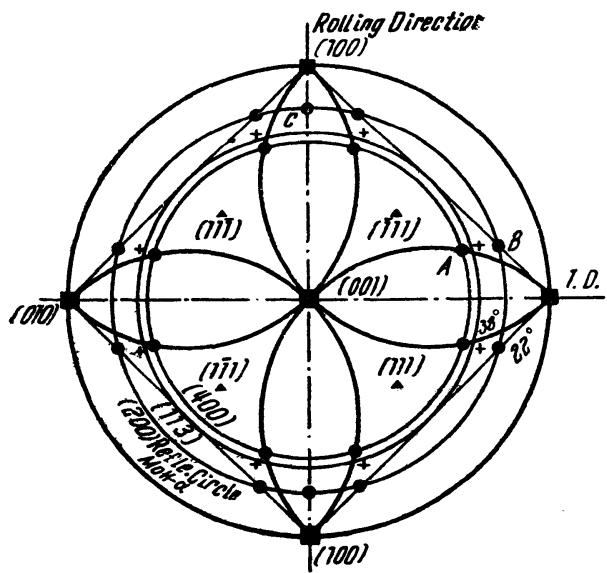

$-\{100\}$ poles of $(001)-[100]$

$+-\{113\}$ poles of $(\mathrm{OO})-[100]$

- $-\{10\}$ poles of the rotated orientations $\dot{A}, \mathrm{~B}$ and $\mathrm{C}$.

Fig. $1\{1 x\}$ and $\{11 j\}$ Poles Figure of Ännealed Copper Sheet, Indicating the $\langle 111\rangle-$ a $1 d\langle 1)\rangle\rangle$ Rotations.

connected with the cubically aligned texture by rotation of $22^{\circ}$ in another direction around [111] axis. Group C corresponds to a rotation of $11^{\circ}$ around [100] axis. Among these rotation relationships, the [111] rotations are in good agreement with those observed by M. L. Kronberg and F. H. Wilson ${ }^{(1)}$ in their research of secondary recrystallization of copper.

into fairly good alignment with the cubically aligned texture by rotations around [111] or [100] axis. Fig. 1 is the $\{113\}$ and $\{100\}$ poles figure indicating these rotation relationships. For convenience of consideration, the (200), (113) and (400) reflection circles are represented in this figure.

As shown in Fig. 1, group A can be connected with the cubically aligned texture by a [111] rotation of about $38^{\circ}$, while group $B$ can be R. D.

$\uparrow$

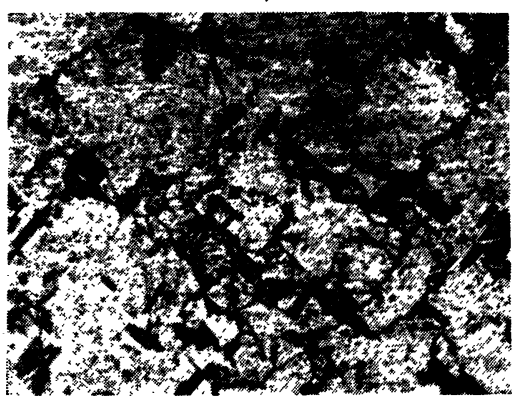

Photo. 6 Microphotograph of Copper Sheet Annealed at $30^{\circ}$ for $10 \mathrm{~min}$, Representing the Twin-bearing Structure

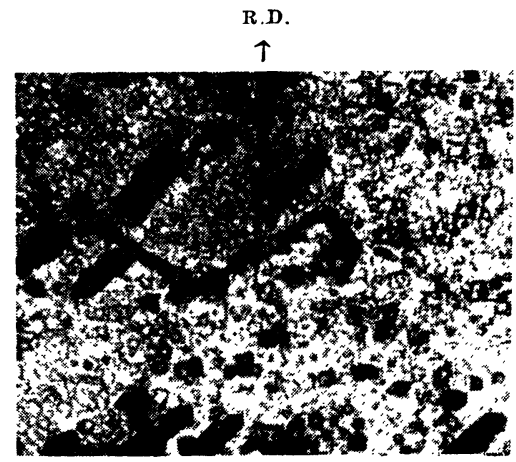

Photo. 7 Micróphotograph of Etching Figure of the Cubically Aligned Structure of Copper Sheets Annealed at $400^{\circ}$ Representing (10))-[001] Structure.

It is clear from Photo. 2 5 that group A ca 1 be found in every annealed specimens, but group B can be found only in two specimens annealed at $200^{\circ}$ and $400^{\circ}$, while group $\mathrm{C}$ comes to appear after annealing at a temperature higher than $600^{\circ}$ In other words, group $\mathrm{B}$ is a low tenperature recrystallization texture and group $\mathrm{C}$ is a high temperature recrystallization texture. 
Photo. 6 illustrates the microstructure of an annealed specimen showing twin-bearing cubically aligned structure. The cubically aligned structure can be clearly seen from the etching figure exhibited in Photo.7. The higher the annealing temperature, the less the number and the area of twins. This phenomenon corresponds to the disapperance of group B.

\section{Discussion}

From the experiment stated above, it has become clear that the oriented growth hypothesis for the origin of recrystallization texture proposed by P. A. Beck and others ${ }^{(2)}$ seems to be inadequate for the interpretation of recrystallization texture of copper. Of course the cubically aligned texture occupies the largest part of an annealed specimen, but the amount of other orientations is not small. The amount and occurence of these other textures vary with temperature. It is difficult to consider that the orientation dependence of the growth velocity of recrystallized grains varies with temperature. The appearance of the recrystallization texture of copper seems to be rationally interpreted by the oriented nucleation hypothesis.

Considering our recent experimental results ${ }^{(3)}$ about the bending of a deformed crystal plane, the dislocation in an active slip plane of face centered cubic metals can be considered as a type represented schematically in Fig. 2. If it is taken into account

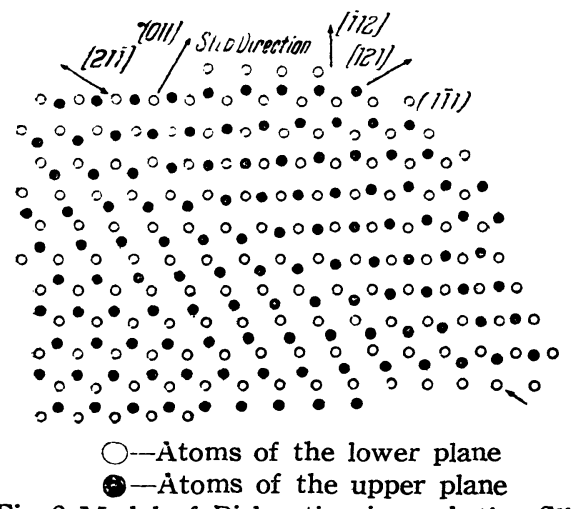

Fig. 2 Model of Dislocation in an Active Slip Plane of Face Centered Cubic Metals

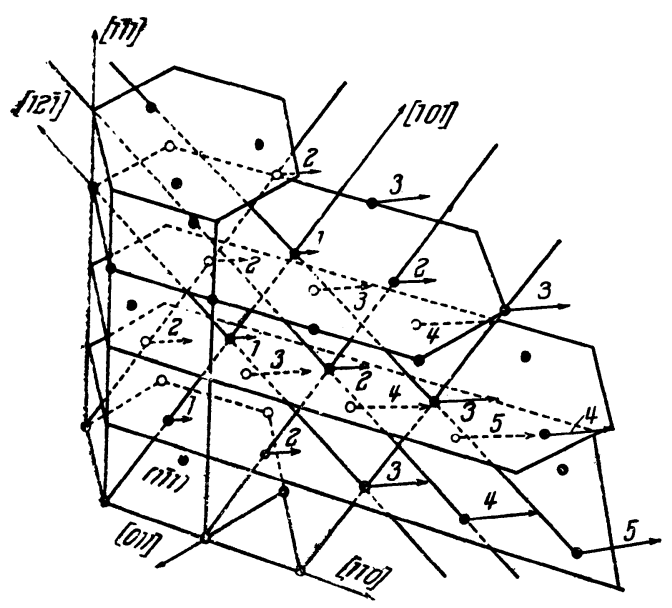

$\rightarrow$ Indicates direction and distance of́ atom displacement.

Fig. 3 Three Dimensional Miodel of the Dislocation Indicated in Fig. 2.

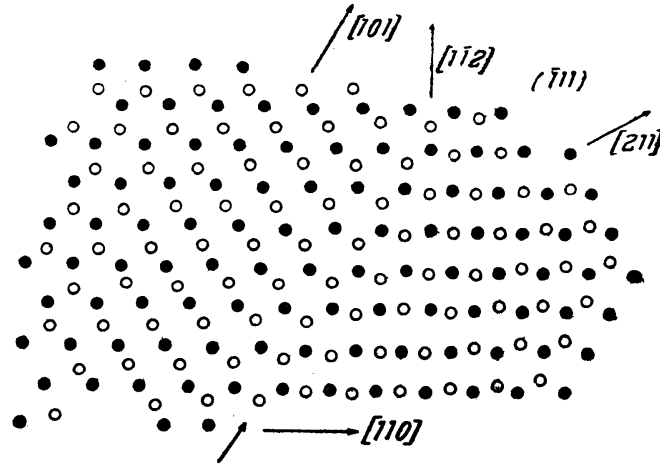

$\bigcirc$-Ảtoms of the lower place

- A toms of the upper plane when the dislocation of active slip plane (111) is the type as shown in Fig. 2 and 3 , local shearing deformation will occur crossing the active slip plane. This phenomenon will be caused by the dislocation in (111) plane indicated in Fig. 4. A composite dislocation is formed by these two types of dislocation.

Fig. 4 Dislocation Model of Face-centered Cubic Metals Expected to Occur by the Components of the Atom Displacement in Dislocation Indicated in Fig. 2.

that the three dimensional model of this dislocation must be as shown in Fig.3, by the component of atom displacement in this dislocation, another type of dislocation as shown in Fig. 4. is expected to appear crossing the active slip plane when deformation bands are formed. However, it is clear that these two types of ideal dislocation can not coexist. Considering the atom displacement in

(2) P. A. Beck, Hsun Hu and P. R. Sperry, J. Applied Physics, 2 (1950), 42 .

(3) I. Gokyu, H. Abe and S. Kohara, Nippon Kinzoku Gakkai-Si, 16 (1952), 529. 
each type of dislocation, a composite dislocation can be considered. In this dislocation the path of atom displacement must be as shown in Fig. 5 .

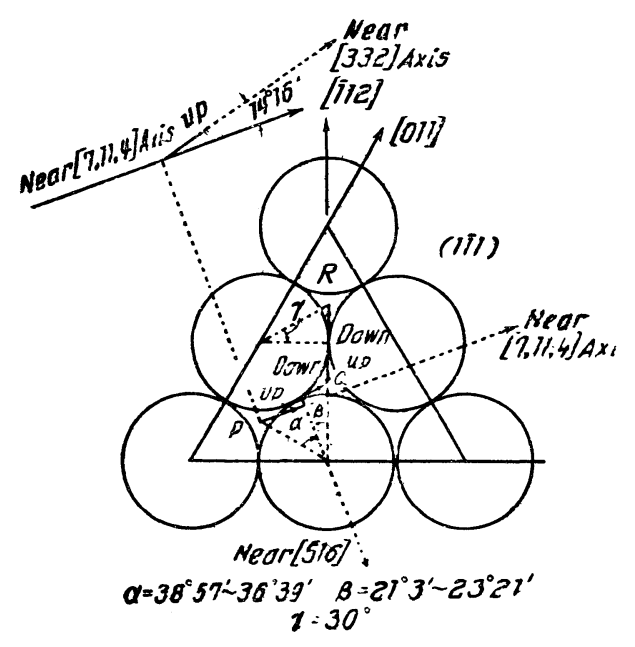

Fig. 5 Path of Ȧtom Displacement in a Composite Dislocation Proposed by the Aiuthers. PQR shows path of the atom displacement in a composite dislocation of face-centered cubic metal on the plane of (1̄i).

This composite dislocation has two points of highest strain and at one of these points the atom rotates about $38^{\circ}$ or $22^{\circ}$ around the.[111] axis. Nucleation will occur at such a point causing a [111] rotation relationship of each lattice part This simple picture seems to offer an explanation of the [111] rotation relationship in copper. The angles of rotation are expected to vary from $38^{\circ}$ $57^{\prime}$ to $36^{\circ} 39^{\prime}$ or from $21^{\circ} 3^{\prime}$ to $23^{\circ} 21^{\prime}$ according to the shape of the potential barrier.

The interpretation stated above supports the idea of nucleation in the classical sence. Recent hypothesis proposed by the authors ${ }^{(4)}$ concerning the origin of the cubically aligned.texture agrees with the idea that the recrystallized grains develope from very small grains which retain ideal lattice during deformation. These two apparantly conflicting viewpoints cannot be distinguished in this investigation, but the idea that a recrystallization texture occurs because only nuclei having a limited range of orientation grow in the deformed matrix seems to be adequate for the origin of primary recrystallization textures.

The P.A.Beck's hypothesis ${ }^{(5)}$ that the formation of the cube orientation component of the annealing texture of aluminium is related to the deformation texture approximately as a [111] rotation of $40^{\circ}$ and that this phenomenon is due to the orientation dependence of the rate of grain boundary migration seems to be inadequate as the [845] axis is not the axis which lies on the (7.12.22) plane and such an orientation proposed by P. A. Beck can not be done crystallographic rotation operation. Our recent researches about the crystal orientation of the individual grain in cold-rolled aluminium and copper show that two types of cold-rolling texture, namely (110,- -[112] and (112)-[111], can be found and the (7.12.22)-[845], (358)-[352] and (2.9.11)-[945] orientations proposed by P. A. Beck are only the geometrical mean orientations of these two types of orientation. In pole figure the scattered distributions of these two types of orientation overlap each other and it looks as if the texture shows such orientations as those proposed by Beck. Furthermore, as J.E. Burke ${ }^{(6)}$ pointed out in his technical note, the fact that the rotation occurs only around a limited number of [111] axis seems to be interpreted as due to the oriented nucleation.

(4) I. Gokyu and H. Abe, Nippon Kinzoku Gakkai--Si, 16 (1952), 534.

(5) P. A. Beck, Hsun Hu and P. R. Sperry, J. Metals, 4 (1952), 76.

(6) J. E. Burke, J. Metals, 4 (1952), 263. 